\title{
Optimal stiffness distribution of mid-storey- isolated buildings considering storey drift and floor acceleration response
}

\author{
M. Kohiyama \& K. Takano \\ Department of System Design Engineering, Keio University, Japan
}

\begin{abstract}
This paper formulates a response prediction formula based on complex modal superposition and frequency response functions of single-degree-of-freedom systems for both storey drift and floor acceleration of a mid-storey-isolated building under the assumption of white noise input. This study verifies that the proposed formula can estimate the seismic response of a building accurately, even if a system includes the overdamped mode, by comparing the same with the results of a time-history analysis. To support the structural design of a midstorey-isolated building, the proposed formula is applied to an optimal design problem, which considers the objective function of acceleration response in a superstructure and constraints on storey drifts. A genetic algorithm is employed to obtain the optimal stiffness distribution and damping coefficient of the isolation layer. This study confirms that the acceleration response of the optimal design is successfully reduced, with other responses remaining within acceptable ranges.

Keywords: mid-storey-isolated buildings, performance-based design, floor acceleration response, complex complete quadratic combination method, overdamped vibration.
\end{abstract}

\section{Introduction}

In Japan, the Building Standard Law regulates seismic design. Two levels of seismic loads are prescribed under this law for preventing structural damage to buildings under moderate and severe ground motions. However, the law only focuses on structural deformation and ultimate resistant force. The acceleration response during an earthquake may lead to serious damages, such as toppling of 
furniture or collapse of ceilings. In fact, such damages have occurred in recent large earthquakes.

A base isolation system is a highly effective approach to reducing the acceleration response of a building, and an increasing number of base-isolated buildings have been constructed in Japan. In addition, a mid-storey-isolation system [1-9] has been devised, and there exist practical examples. However, a design scheme which considers both structural deformation and acceleration response has not been proposed so far.

In this study, first, a response prediction formula based on complex modal superposition and frequency response functions of single-degree-of-freedom (SDOF) systems for both storey drift and floor acceleration is formulated under the assumption of white noise input. The derived formula is extended from a response spectrum method for a non-classically damped system proposed by Igusa et al. [10] and Zhou et al. [11] considering overdamped modes, and is expressed in a simpler and more uniform form than that proposed by Song et al. [12]. The estimation accuracy is then examined by comparing the results of a time-history analysis using a mid-storey-isolated building model. Finally, the proposed formula is applied to the optimal design of a mid-storey-isolated building, and the seismic performance of the optimal design is verified.

\section{Response prediction formula considering overdamped modes}

We focus on the mass shear spring model of an $N$-storey building, which responds in the linear-elastic range. When the system is excited by ground motion, its state-space equation of motion is described as follows:

$$
\dot{\mathbf{x}}_{\mathrm{ss}}+\mathbf{B}_{\mathrm{ss}} \mathbf{x}_{\mathrm{ss}}=\mathbf{g}_{\mathrm{ss}} \ddot{x}_{\mathrm{g}},
$$

where

$$
\mathbf{x}_{\mathrm{ss}}=\left\{\begin{array}{l}
\dot{\mathbf{x}} \\
\mathbf{x}
\end{array}\right\}, \mathbf{B}_{\mathrm{ss}}=\left[\begin{array}{cc}
\mathbf{M}^{-1} \mathbf{C} & \mathbf{M}^{-1} \mathbf{K} \\
-\mathbf{I} & \mathbf{O}
\end{array}\right], \mathbf{g}_{\mathrm{ss}}=\left\{\begin{array}{c}
-\mathbf{1} \\
\mathbf{0}
\end{array}\right\} .
$$

$\mathbf{M}, \mathbf{C}$ and $\mathbf{K}$ are the mass, damping and stiffness matrices, respectively. The $N$-component vector $\mathbf{x}(t)$ is composed of the displacement of the mass point $i$ relative to the ground, $x_{i}(t) ; \ddot{x}_{\mathrm{g}}(t)$ represents ground acceleration, and $\mathbf{1}$ is an $N$ component column vector in which all elements are 1.

\subsection{Overdamped modes}

The equation of motion for free vibration, i.e. $\dot{\mathbf{x}}_{\mathrm{ss}}+\mathbf{B}_{\mathrm{ss}} \mathbf{x}_{\mathrm{ss}}=\mathbf{0}$, induces the following eigenvalue equation and characteristic equation:

$$
\left(\Omega \mathbf{I}+\mathbf{B}_{\mathrm{ss}}\right) \boldsymbol{\varphi}_{\mathrm{ss}}=\mathbf{0} \text { and } \operatorname{det}\left(\Omega \mathbf{I}+\mathbf{B}_{\mathrm{ss}}\right)=0,
$$


where $\Omega$ is the eigenvalue, and $\varphi_{\mathrm{ss}}$ is the right eigenvector. The $n^{\text {th }}$ mode vector $\varphi_{\text {ss } n}$ can be expressed by the $n^{\text {th }}$ eigenvalue $\Omega_{n}$ and the $n^{\text {th }}$ displacement mode vector $\varphi_{n}$ as follows:

$$
\varphi_{\mathrm{ss} n}=\left\{\Omega_{n} \varphi_{n}{ }^{T} \varphi_{n}{ }^{T}\right\}^{T} .
$$

Let mode numbers $n=1,2, \ldots, M$ represent underdamped vibration and $n=M$ $+1, M+2, \ldots, N$ overdamped vibration. We obtain $M$ complex conjugate pairs of $\Omega$ and $\varphi_{\text {ss }}$ related to underdamped modes, and $(N-M)$ real value pairs of $\Omega$ and $\varphi_{\mathrm{ss}}$ related to overdamped modes. The state vector $\mathbf{x}_{\mathrm{ss}}$ is expressed by the modal coordinate $\mathbf{q}_{\mathrm{ss}}$ as follows:

where

$$
\mathbf{x}_{\mathrm{ss}}=\Phi_{\mathrm{ss}} \mathbf{q}_{\mathrm{ss}}
$$

$$
\begin{aligned}
\Phi_{\mathrm{ss}}= & {\left[\varphi_{\mathrm{ss} 1} \varphi_{\mathrm{ss} 2} \cdots \varphi_{\mathrm{ss} M} \varphi_{\mathrm{ss} M+1} \varphi_{\mathrm{ss} M+2} \cdots \varphi_{\mathrm{ss} N}\right.} \\
& \left.\varphi_{\mathrm{ss} 1}^{*} \varphi_{\mathrm{ss} 2}^{*} \cdots \varphi_{\mathrm{ss} M}^{*} \varphi_{\mathrm{ss} N+M+1} \varphi_{\mathrm{ss} N+M+2} \cdots \varphi_{\mathrm{ss} 2 N}\right]
\end{aligned}
$$

The asterisks indicate complex conjugates, and $\varphi_{\mathrm{ss} n+N}=\varphi_{\mathrm{ss} n}^{*}(n=1,2, \ldots$, $M)$ for underdamped modes. The pairs of $\varphi_{\mathrm{ss} n}$ and $\varphi_{\mathrm{ss} n+N}(n=M+1, M+2, \ldots$, $N)$ are associated with overdamped modes.

Using the modal matrix, the equation of motion is decoupled as follows:

$$
\begin{gathered}
\Phi_{\mathrm{ss}}^{-1} \Phi_{\mathrm{ss}} \dot{\mathbf{q}}_{\mathrm{ss}}+\Phi_{\mathrm{ss}}^{-1} \mathbf{B}_{\mathrm{ss}} \Phi_{\mathrm{ss}} \mathbf{q}_{\mathrm{ss}}=\Phi_{\mathrm{ss}}^{-1} \mathbf{g}_{\mathrm{ss}} \ddot{x}_{\mathrm{g}} \Leftrightarrow \dot{\mathbf{q}}_{\mathrm{ss}}-\Omega \mathbf{q}_{\mathrm{ss}}=\Gamma \ddot{x}_{\mathrm{g}} \\
\Leftrightarrow \dot{q}_{\mathrm{ss} n}(t)-\Omega_{n} q_{\mathrm{ss} n}(t)=\Gamma_{n} \ddot{x}_{\mathrm{g}}(t) \quad(n=1,2, \ldots, 2 N)
\end{gathered}
$$

where $\Gamma_{n}$ is the $n^{\text {th }}$ element of the $2 N$-component vector $\Gamma=\Phi_{\mathrm{ss}}^{-1} \mathbf{g}_{\mathrm{ss}}$ and is referred to as participation factor of the $n^{\text {th }}$ mode. The diagonal matrix $\Omega=\operatorname{diag}\left(\Omega_{n}\right)=-\boldsymbol{\Phi}_{\mathrm{ss}}^{-1} \mathbf{B}_{\mathrm{ss}} \boldsymbol{\Phi}_{\mathrm{ss}}$ has $\Omega_{n}$ in its $(n, n)$ element. For underdamped modes, $\Omega_{n}=-\zeta_{n} \omega_{n}+\mathrm{i} \omega_{n} \sqrt{1-\zeta_{n}^{2}}$ and $\Omega_{n+N}=-\zeta_{n} \omega_{n}-\mathrm{i} \omega_{n} \sqrt{1-\zeta_{n}^{2}}$ are complex conjugate pairs, where $\zeta_{n}$ and $\omega_{n}$ are the damping factor and the natural circular frequency of the $n^{\text {th }}$ mode, respectively, and $\mathrm{i}$ is the imaginary unit. For overdamped modes, $\Omega_{n}=\Omega_{n}^{+}=-\zeta_{n} \omega_{n}+\omega_{n} \sqrt{\zeta_{n}^{2}-1}$ and $\Omega_{n+N}=\Omega_{n}^{-}=-\zeta_{n} \omega_{n}$ $-\omega_{n} \sqrt{\zeta_{n}^{2}-1}$ are real values. These parameters have the following relations:

For underdamped modes $(n=1,2, \ldots, M)$,

$$
\begin{gathered}
\omega_{n}=\operatorname{sgn}\left(\operatorname{Im} \Omega_{n}\right)\left|\Omega_{n}\right|, \omega_{\mathrm{d} n}=\omega_{n} \sqrt{1-\zeta_{n}^{2}}=\operatorname{Im} \Omega_{n} \\
\text { and } \zeta_{n}=-\operatorname{Re}\left(\Omega_{n}\right) /\left|\Omega_{n}\right| .
\end{gathered}
$$

For overdamped modes $(n=M+1, M+2, \ldots, N)$,

$$
\omega_{n}=\sqrt{\Omega_{n}^{+} \Omega_{n}^{-}}, \omega_{\mathrm{d} n}=\omega_{n} \sqrt{\zeta_{n}^{2}-1}
$$


194 Earthquake Resistant Engineering Structures IX

$$
\text { and } \zeta_{n}=\sqrt{\frac{\psi}{\psi-1}} \text { where } \psi=\left(\frac{\Omega_{n}^{+}+\Omega_{n}^{-}}{\Omega_{n}^{+}-\Omega_{n}^{-}}\right)^{2}
$$

\subsection{Modal superposition}

The solution of eqn. (11) is given by

$$
q_{\mathrm{ss} n}(t)=\Gamma_{n} \int_{0}^{t} \ddot{x}_{\mathrm{g}}(\tau) \exp \left(\Omega_{n}(t-\tau)\right) \mathrm{d} \tau .
$$

By substituting eqn. (19) in eqn. (8),

$$
\begin{aligned}
& \mathbf{x}_{\mathrm{ss}}=\sum_{n=1}^{N} \Gamma_{n} \boldsymbol{\varphi}_{\mathrm{ss} n} \int_{0}^{t} \ddot{x}_{\mathrm{g}}(\tau) \exp \left(\Omega_{n}(t-\tau)\right) \mathrm{d} \tau \\
& =2 \operatorname{Re} \sum_{n=1}^{M} \Gamma_{n}\left\{\begin{array}{c}
\Omega_{n} \varphi_{n} \\
\varphi_{n}
\end{array}\right\} \int_{0}^{t} \ddot{x}_{\mathrm{g}}(\tau) \exp \left(\Omega_{n}(t-\tau)\right) \mathrm{d} \tau \\
& +\sum_{n=M+1}^{N}\left[\Gamma_{n}\left\{\begin{array}{c}
\Omega_{n} \varphi_{n} \\
\varphi_{n}
\end{array}\right\} \int_{0}^{t} \ddot{x}_{\mathrm{g}}(\tau) \exp \left(\Omega_{n}(t-\tau)\right) \mathrm{d} \tau\right. \\
& \left.+\Gamma_{n+N}\left\{\begin{array}{c}
\Omega_{n+N} \varphi_{n+N} \\
\varphi_{n+N}
\end{array}\right\} \int_{0}^{t} \ddot{x}_{\mathrm{g}}(\tau) \exp \left(\Omega_{n+N}(t-\tau)\right) \mathrm{d} \tau\right]
\end{aligned}
$$

and using the $n^{\text {th }}$ mode participation vector $\mathbf{b}_{n}=\Gamma_{n} \boldsymbol{\varphi}_{n}$, the lower half of $\mathbf{x}_{\mathrm{ss}}$ gives the displacement vector $\mathbf{x}$ as follows:

$$
\begin{aligned}
& \mathbf{x}= 2 \operatorname{Re} \sum_{n=1}^{M} \mathbf{b}_{n} \int_{0}^{t} \ddot{x}_{\mathrm{g}}(\tau) \exp \left(\Omega_{n}(t-\tau)\right) \mathrm{d} \tau \\
&++\sum_{n=M+1}^{N}\left[\mathbf{b}_{n} \int_{0}^{t} \ddot{x}_{\mathrm{g}}(\tau) \exp \left(\Omega_{n}(t-\tau)\right) \mathrm{d} \tau\right. \\
&\left.\quad+\mathbf{b}_{n+N} \int_{0}^{t} \ddot{x}_{\mathrm{g}}(\tau) \exp \left(\Omega_{n+N}(t-\tau)\right) \mathrm{d} \tau\right] \\
&=\sum_{n=1}^{N}\left(\mathbf{a}_{n} h_{n}(t)+\mathbf{c}_{n} \dot{h}_{n}(t)\right)
\end{aligned}
$$

where

$$
\begin{aligned}
& \mathbf{a}_{n}= \begin{cases}2 \operatorname{Re}\left(\Omega_{n}^{*} \mathbf{b}_{n}\right) & (1 \leq n \leq M) \\
\Omega_{n}^{-} \mathbf{b}_{n}+\Omega_{n}^{+} \mathbf{b}_{n+N} & (M+1 \leq n \leq N)\end{cases} \\
& \text { and } \mathbf{c}_{n}= \begin{cases}-2 \operatorname{Re} \mathbf{b}_{n} & (1 \leq n \leq M) \\
-\mathbf{b}_{n}-\mathbf{b}_{n+N} & (M+1 \leq n \leq N)\end{cases}
\end{aligned}
$$

and $h_{n}(t)$ is the displacement response of an SDOF system with natural circular frequency $\omega_{n}$ and damping factor $\zeta_{n}$ under ground motion. Eqns. (21)-(23) are the rigorous forms of modal superposition considering overdamped modes. 


\subsection{Response prediction formula considering overdamped modes}

Next, following the response spectrum methods for a non-classically damped system, a response prediction formula for storey drift and floor acceleration is formulated under a white noise input considering overdamped modes. Using eqn. (21), the autocorrelation of $x_{i}$ is expressed as follows:

$$
\begin{aligned}
R_{x_{i} x_{i}}(\tau) & =E\left[x_{i}(t) x_{i}(t+\tau)\right] \\
& =\sum_{m=1}^{N} \sum_{n=1}^{N} E\left[\left(a_{i, m} h_{m}(t)+c_{i, m} \dot{h}_{m}(t)\right)\left(a_{i, n} h_{n}(t+\tau)+c_{i, n} \dot{h}_{n}(t+\tau)\right)\right]
\end{aligned}
$$

where $a_{i, n}$ and $c_{i, n}$ are the $i^{\text {th }}$ components of $\mathbf{a}_{n}$ and $\mathbf{c}_{n}$, respectively. The one-sided power spectral density (PSD) of $x_{i}$ is given by

$$
\begin{gathered}
G_{x_{i} x_{i}}(\omega)=\sum_{m=1}^{N} \sum_{n=1}^{N}\left[a_{i, m} a_{i, n}+\mathrm{i} \omega\left(a_{i, m} c_{i, n}-a_{i, n} c_{i, m}\right)+\omega^{2} c_{i, m} c_{i, n}\right] \\
\cdot G_{0}(\omega) H_{m}^{*}(\omega) H_{n}(\omega)
\end{gathered}
$$

where

$$
H_{n}(\omega)=-\frac{1}{\omega_{n}^{2}-\omega^{2}+2 \mathrm{i} \zeta_{n} \omega_{n} \omega},
$$

which is the frequency response function of the SDOF system with the $n^{\text {th }}$ mode characteristics, and $G_{0}(\omega)$ is the PSD of the ground acceleration.

The square power of $x_{i}$ is derived as follows:

$$
\begin{aligned}
& E\left[x_{i}^{2}(t)\right]=R_{x_{i} x_{i}}(0)=\int_{0}^{\infty} G_{x_{i} x_{i}}(\omega) \mathrm{d} \omega \\
= & \sum_{m=1}^{N} \sum_{n=1}^{N}\left[a_{i, m} a_{i, n} \operatorname{Re} \lambda_{0, m n}-\left(a_{i, m} c_{i, n}-a_{i, n} c_{i, m}\right) \operatorname{Im} \lambda_{1, m n}+c_{i, m} c_{i, n} \operatorname{Re} \lambda_{2, m n}\right]
\end{aligned}
$$

where

$$
\lambda_{l, m n}=\int_{0}^{\infty} \omega^{l} G_{0}(\omega) H_{m}(\omega) H_{n}^{*}(\omega) \mathrm{d} \omega .
$$

Igusa et al. [10] provide the cross-spectral moments $\lambda_{l, m n}$ for response to the white-noise input $G_{0}$.

Introducing the modal correlation coefficients $\rho_{m n}^{\mathrm{DD}}, \rho_{m n}^{\mathrm{vD}}$ and $\rho_{m n}^{\mathrm{VV}}$ defined by Zhou et al. [11], eqn. (27) gives

$$
\begin{gathered}
E\left[x_{i}^{2}(t)\right]=\sum_{m=1}^{N} \sum_{n=1}^{N}\left(\rho_{m n}^{\mathrm{DD}} a_{i, m} a_{i, n}-2 \rho_{m n}^{\mathrm{VD}} c_{i, m} a_{i, n} \omega_{m}+\rho_{m n}^{\mathrm{VV}} c_{i, m} c_{i, n} \omega_{m} \omega_{n}\right) \\
\cdot \sqrt{\operatorname{Re} \lambda_{0, m m}} \sqrt{\operatorname{Re} \lambda_{0, n n}}
\end{gathered}
$$

Introducing the parameter $\Lambda_{n}=\sqrt{\operatorname{Re} \lambda_{0, n n}}$, which is the standard deviation of the displacement of the $n^{\text {th }}$ mode SDOF system under white noise input $G_{0}$, the root-mean-square (RMS) value of $x_{i}$ is given by 


$$
D_{i}^{\mathrm{RMS}}=\sqrt{\sum_{m=1}^{N} \sum_{n=1}^{N}\left(\rho_{m n}^{\mathrm{DD}} a_{i, m} a_{i, n}-2 \rho_{m n}^{\mathrm{VD}} c_{i, m} a_{i, n} \omega_{m}+\rho_{m n}^{\mathrm{VV}} c_{i, m} c_{i, n} \omega_{m} \omega_{n}\right) \Lambda_{m} \Lambda_{n}} .
$$

Similarly, the RMS value of the storey drift in $i^{\text {th }}$ storey is given by

$$
\theta_{i}^{\mathrm{RMS}}=\frac{1}{h_{\mathrm{b} i}} \sqrt{\sum_{m=1}^{N} \sum_{n=1}^{N}\left(\rho_{m n}^{\mathrm{DD}} \widetilde{a}_{i, m} \widetilde{a}_{i, n}-2 \rho_{m n}^{\mathrm{VD}} \widetilde{c}_{i, m} \widetilde{a}_{i, n} \omega_{m}+\rho_{m n}^{\mathrm{VV}} \widetilde{c}_{i, m} \widetilde{c}_{i, n} \omega_{m} \omega_{n}\right) \Lambda_{m} \Lambda_{n}}
$$

where

$$
\widetilde{a}_{i, n}=a_{i, n}-a_{i-1, n} \text { and } \widetilde{c}_{i, n}=c_{i, n}-c_{i-1, n}
$$

and $a_{0, n}=c_{0, n}=0$. The parameter $h_{\mathrm{b} i}$ is the $i^{\text {th }}$ storey height.

The absolute acceleration response is given by the equation of motion:

$$
\ddot{\mathbf{x}}(t)+\mathbf{1} \ddot{x}_{\mathrm{g}}(t)=-\mathbf{M}^{-1} \mathbf{K} \mathbf{x}(t)-\mathbf{M}^{-1} \mathbf{C} \dot{\mathbf{x}}(t) .
$$

By substituting eqn. (21) in (34),

$$
\begin{aligned}
& \ddot{\mathbf{x}}(t)+1 \ddot{x}_{\mathrm{g}}(t)=2 \operatorname{Re} \sum_{n=1}^{M} \hat{\mathbf{b}}_{n} \int_{0}^{t} \ddot{x}_{\mathrm{g}}(\tau) \exp \left(\Omega_{n}(t-\tau)\right) \mathrm{d} \tau \\
&+\sum_{n=N+1}^{N}\left[\hat{\mathbf{b}}_{n} \int_{0}^{t} \ddot{x}_{\mathrm{g}}(\tau) \exp \left(\Omega_{n}(t-\tau)\right) \mathrm{d} \tau\right. \\
&\left.+\hat{\mathbf{b}}_{n+N} \int_{0}^{t} \ddot{x}_{\mathrm{g}}(\tau) \exp \left(\Omega_{n+N}(t-\tau)\right) \mathrm{d} \tau\right]
\end{aligned}
$$

where

$$
\hat{\mathbf{b}}_{n}=-\left(\mathbf{M}^{-1} \mathbf{K}+\Omega_{n} \mathbf{M}^{-1} \mathbf{C}\right) \mathbf{b}_{n} .
$$

Because eqn. (35) is the same as eqn. (21), the RMS value of the acceleration response is deduced from eqn. (30) by substituting $\hat{\mathbf{b}}_{n}$ for $\mathbf{b}_{n}$ :

$$
A_{i}^{\mathrm{RMS}}=\sqrt{\sum_{m=1}^{N} \sum_{n=1}^{N}\left(\rho_{m n}^{\mathrm{DD}} \hat{a}_{i, m} \hat{a}_{i, n}-2 \rho_{m n}^{\mathrm{VD}} \hat{c}_{i, m} \hat{a}_{i, n} \omega_{m}+\rho_{m n}^{\mathrm{VV}} \hat{c}_{i, m} \hat{c}_{i, n} \omega_{m} \omega_{n}\right) \Lambda_{m} \Lambda_{n}}
$$

where $\hat{a}_{i, n}$ and $\hat{c}_{i, n}$ are the $i^{\text {th }}$ component of the following vectors, respectively:

$$
\begin{gathered}
\hat{\mathbf{a}}_{n}= \begin{cases}2 \operatorname{Re}\left(\Omega_{n}^{*} \hat{\mathbf{b}}_{n}\right) & (1 \leq n \leq M) \\
\Omega_{n}^{-} \hat{\mathbf{b}}_{n}+\Omega_{n}^{+} \hat{\mathbf{b}}_{n+N} & (M+1 \leq n \leq N)\end{cases} \\
\text { and } \hat{\mathbf{c}}_{n}=\left\{\begin{array}{ll}
-2 \operatorname{Re} \hat{\mathbf{b}}_{n} & (1 \leq n \leq M) \\
-\hat{\mathbf{b}}_{n}-\hat{\mathbf{b}}_{n+N} & (M+1 \leq n \leq N)
\end{array} .\right.
\end{gathered}
$$

\section{Verification of response prediction formula}

To examine the accuracy of the proposed response prediction formula, a 16storey-building model was considered, in which an isolation system composed of 
an isolator bearing and a damper was inserted in the fifth storey. The building was modelled as a mass-spring system, with a mass of $1 \times 10^{6} \mathrm{~kg}$ for each mass point.

The height of each storey was assumed to be $3.5 \mathrm{~m}$, and the total height of the model was $56 \mathrm{~m}$. The stiffness distribution of normal storeys (those without the isolator) was calculated on the basis of the Ai distribution of the Building Standard Law of Japan for a building with a fundamental natural period of 1.68 $\mathrm{s}$, neglecting the isolation system. Stiffness proportional damping $\mathbf{C}=\left(2 \zeta_{1} / \omega_{1}\right)$ $\mathbf{K}$ was assumed with a damping factor $\zeta_{1}$ of 0.02 for the normal storeys, and the proportionality factor between the damping coefficient and stiffness was maintained during the optimization process. The stiffness of the isolator was assigned so that the natural period of an SDOF system considering the mass of the superstructure (isolation period) is $3 \mathrm{~s}$. The damping coefficient of the isolation system was given as $3 \times 10^{7} \mathrm{Ns} / \mathrm{m}$. It is supposed that the system responses are in the linear elastic range.

Fig. 1 shows the modal properties of the fifth-storey-isolated building. Modal participation vectors $\mathbf{b}_{n}$ are depicted by the amplitude and phase distributions. An overdamped mode appears in the fourth mode, and a pair of real value vectors is shown in the graph rather than the amplitude and phase. Fig. 2 shows the modal participation vectors of the absolute acceleration response $\hat{\mathbf{b}}_{n}$. The vectors are large even in higher modes, especially in the lower part of the building.

The RMS response values estimated by the proposed formula were compared with those calculated by the time-history analysis in fig. 3. The white-noise
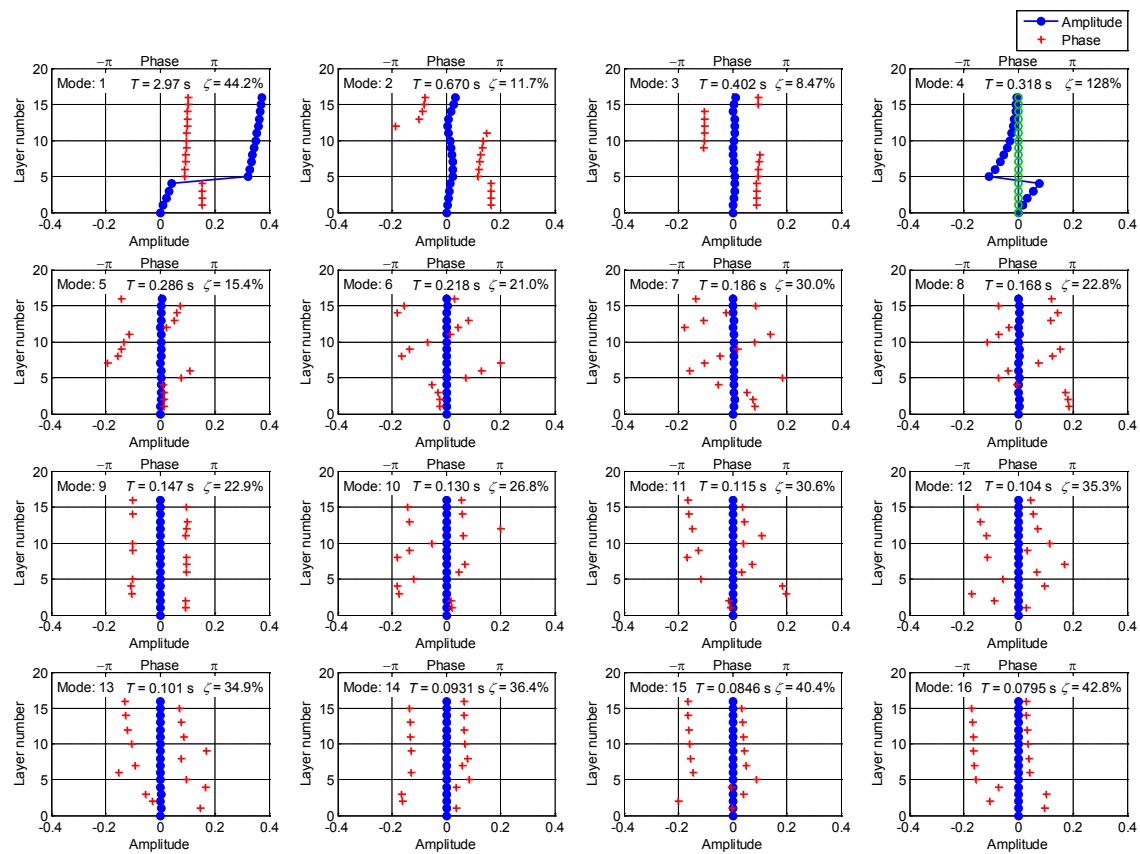

Figure 1: Modal participation vectors of a fifth-storey-isolated building. 

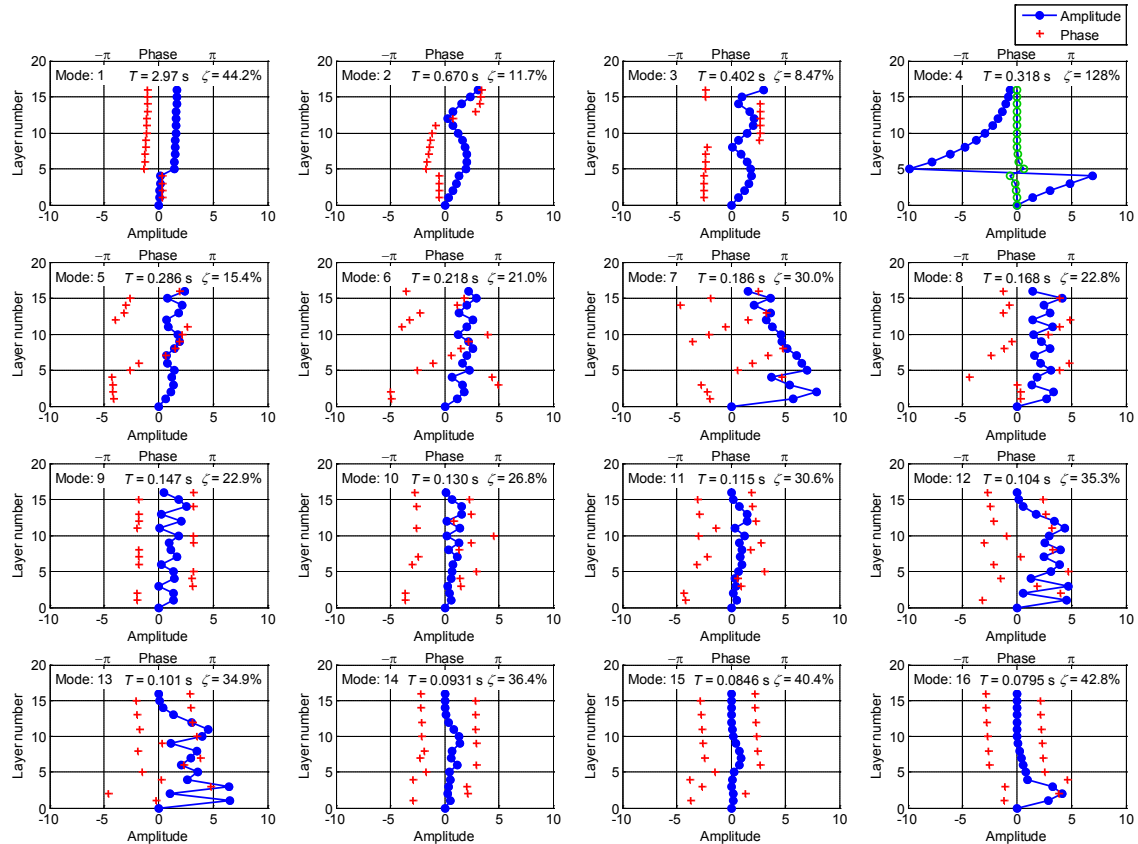

Figure 2: Modal participation vectors of absolute acceleration response of a fifth-storey-isolated building.

ground acceleration with $G_{0}=1.19 \times 10^{-5} \mathrm{~m}^{2} \cdot \mathrm{s}^{-3}$ is generated by a random Fourier phase spectrum and a constant Fourier amplitude spectrum. The integration time interval and duration of input motion are $0.001 \mathrm{~s}$ and $300 \mathrm{~s}$, respectively.

Fairly good agreements are confirmed in fig. 3. The storey drift in the isolation storey was omitted on the graph because this value was considerably larger than the storey drift in other storeys. A good agreement up to three digits
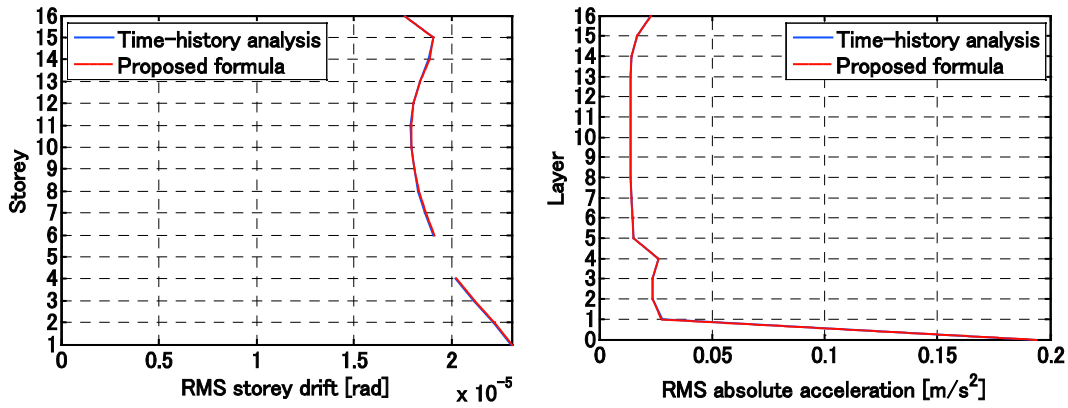

Figure 3: Comparison of RMS response values between the proposed formula and a time-history analysis; left: storey drift and right: absolute acceleration response. 
was also confirmed with respect to the deformation of the isolation storey, which was $0.00148 \mathrm{~m}$. Note that the RMS absolute acceleration of the ground level is also shown in fig. 3 for reference purposes.

\section{Optimal design of a mid-storey-isolated building}

The proposed response prediction method was applied to the structural optimization of a mid-storey-isolated building. The proposed method enables us to avoid performing a time-history analysis, which has a higher computational cost when long-duration ground motions are to be input. A genetic algorithm was employed for optimization, and the $1000^{\text {th }}$ generation was selected as the termination condition for the optimization. The model described in the previous section was used as the initial design. The following objective function (maximum RMS absolute acceleration in the superstructure) and constraints were introduced in the optimization process:

$$
\text { Minimize } J\left(k_{1}, k_{2}, \ldots, k_{16}, c_{5}\right)=\max _{i \geq 5}\left(E\left[\left(\ddot{x}_{i}+\ddot{x}_{\mathrm{g}}\right)^{2}\right]\right)
$$

subject to

$$
\begin{gathered}
k_{1} \geq k_{2} \geq k_{3} \geq k_{4} \geq k_{6} \geq k_{7} \geq \ldots \geq k_{16} \\
\sum_{i, i \neq 5} k_{i} \leq \sum_{i, i \neq 5} k_{i \text { ini }} \\
k_{5}>k_{5 \text { ini }} \\
\max _{i, i \neq 5} \theta_{i \mathrm{RMS}}<1 / 600 \mathrm{rad} \\
\text { and } h_{\mathrm{b} 5} \theta_{5 \mathrm{RMS}}<0.1 \mathrm{~m}
\end{gathered}
$$

where $k_{i}$ and $c_{i}$ represent the stiffness and damping coefficient of the $i^{\text {th }}$ storey, respectively. The subscript 'ini' refers to the initial model. The constraints (41) to (45) provide the monotonically decreasing stiffness, maximum total stiffness, minimum isolator stiffness, storey deformation restriction, and isolator deformation restriction, respectively.

The optimization results are shown in fig. 4 (stiffness and damping coefficient distributions), fig. 5 (RMS response values), fig. 6 (modal participation vector) and fig. 7 (modal participation vector of absolute acceleration response).
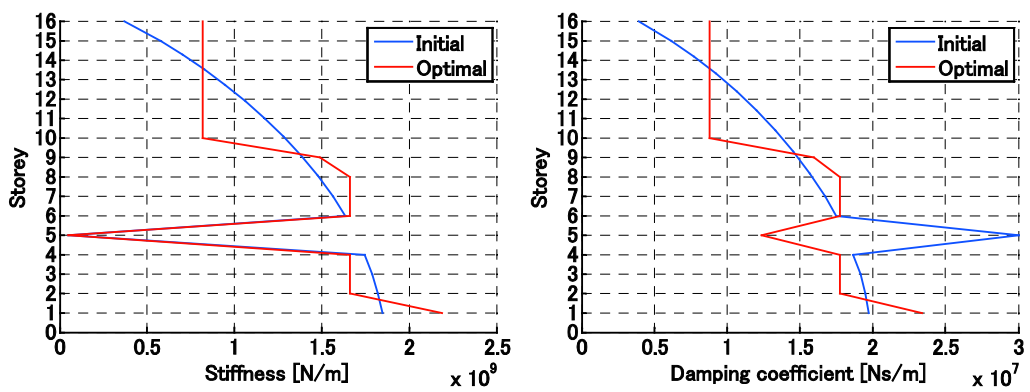

Figure 4: Comparison of stiffness and damping coefficient distributions between the initial and optimal designs. 

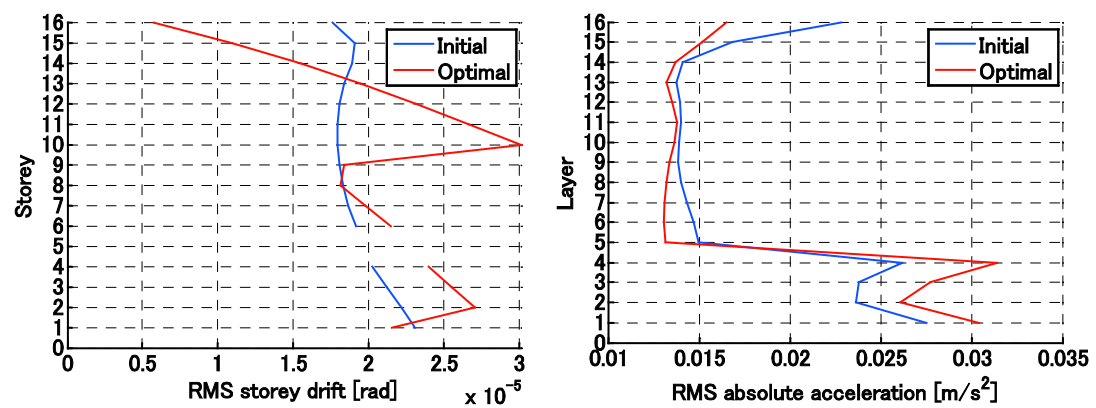

Figure 5: Comparison of RMS response values between the initial and optimal designs; left: storey drift and right: absolute acceleration response.
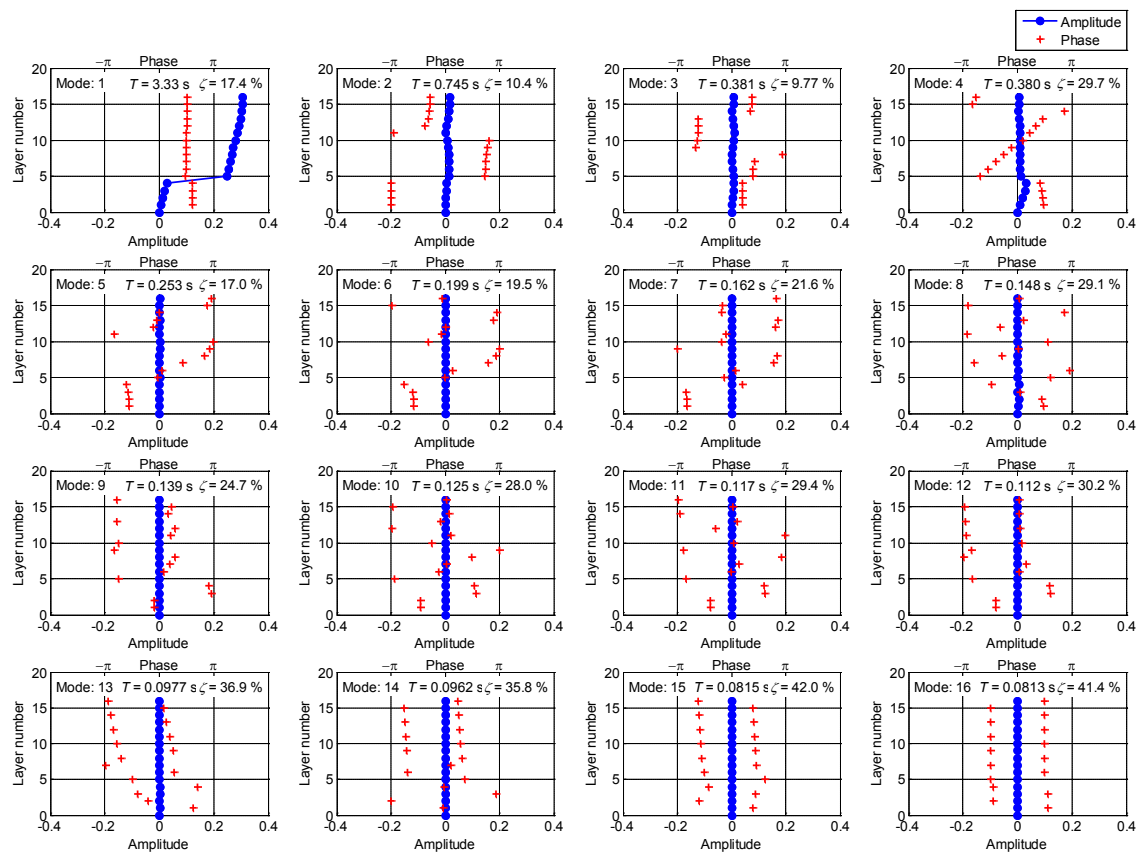

Figure 6: Modal participation vectors of the optimal design.

It is noteworthy that the stiffness of the storeys above and below the isolation storey coincide (fig. 4 left), and the damping coefficient of the isolation storey was reduced in the optimal design (fig. 4 right), which consequently resulted in zero overdamped modes (figs. 6 and 7). The absolute acceleration response of the superstructure was successfully reduced, especially in the upper storeys (fig. 5 right). Both absolute acceleration response of the substructure and storey drifts in many storeys increased in the optimal design (fig. 5), but both remained within acceptable ranges. It is demonstrated that the proposed response 
prediction formula can help find a suitable structural design considering both structural deformation and acceleration response.
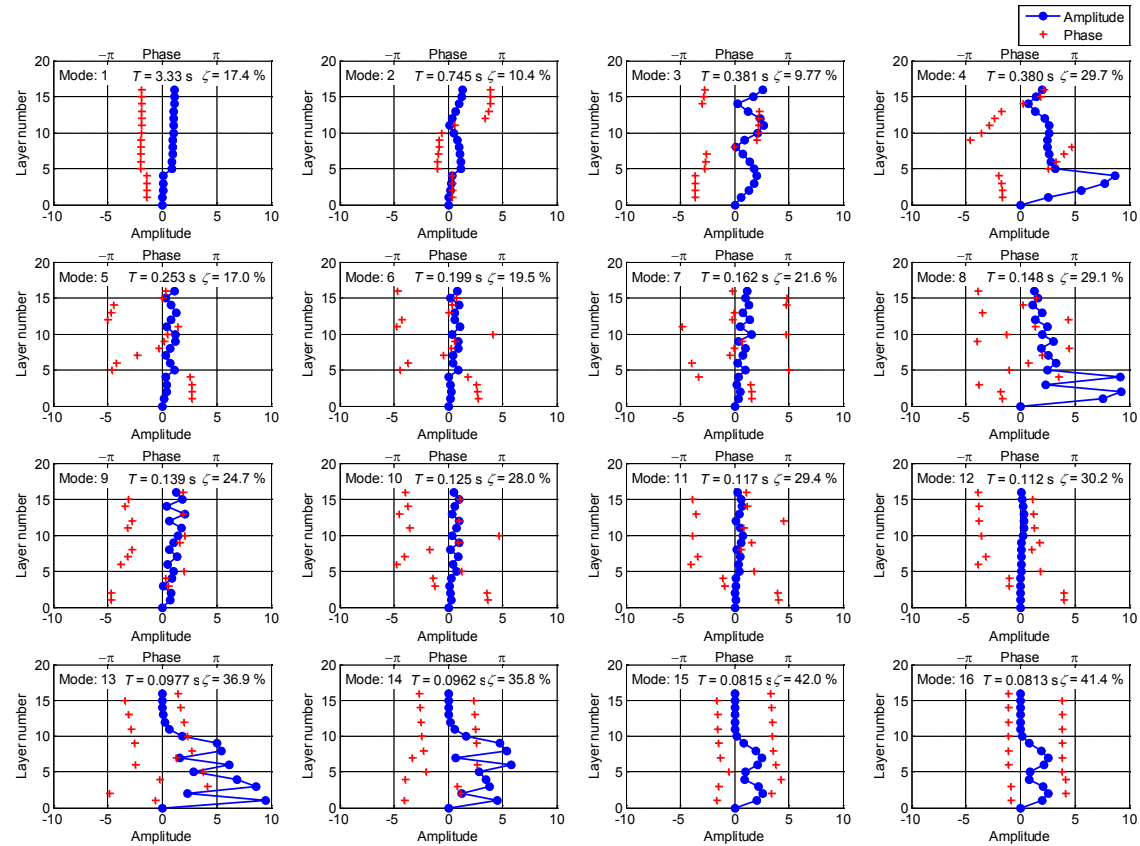

Figure 7: Modal participation vectors of the absolute acceleration response of the optimal design.

\section{Conclusions}

This paper proposes a formula based on complex modal superposition and frequency response functions of single-degree-of-freedom systems for the estimation of storey drift and floor acceleration under the assumption of white noise input. The formula can estimate the seismic response of a building accurately, even if a system includes the overdamped mode. The proposed formula was applied to the structural optimization of a mid-storey-isolated building, and it was verified that the absolute acceleration of the superstructure could be successfully reduced, with other responses remaining within acceptable ranges.

With respect to input ground motion, future studies are required to investigate the applicability of the proposed formula to seismic ground motion, rather than white noise. The seismic performance of an optimal design under seismic ground motion also needs to be examined.

\section{Acknowledgement}

This study was supported by a JSPS KAKENHI Grant-in-Aid for Scientific Research (B) Grant Number 24360230. 


\section{References}

[1] Ziyaeifar, M. and Noguchi H., Partial mass isolation in tall buildings. Earthquake Engineering \& Structural Dynamics, 27(1), pp. 49-65, 1998.

[2] Ogura K., Takayama M., Tsujita O., Kimura Y. and Wada A., Seismic response of mid-story isolated buildings. Journal of Structural and Construction Engineering, Transaction of Architectural Institute of Japan (AIJ), (516), pp. 99-104, 1999 (in Japanese).

[3] Murakami, K., Kitamura, H. and Matsushima, Y., The prediction for seismic Responses of the two-mass model with the mid-story isolation system. Journal of Structural and Construction Engineering, Transaction of AIJ, (549), pp. 51-58, 2001 (in Japanese).

[4] Kobayashi, M. and Koh, T., Earthquake response prediction and aseismic performance of mid-story isolated system. Journal of Structural and Construction Engineering, Transaction of AIJ, (558), pp. 109-116, 2002 (in Japanese).

[5] Kobayashi, M., Izawa, Y. and Koh, T., The prediction method of earthquake responses on mid-story isolated system considering modal coupling effect: Earthquake response prediction and aseismic performance of mid-story isolated system Part 2. Journal of Structural and Construction Engineering, Transaction of AIJ, (572), pp. 73-80, 2003 (in Japanese).

[6] Kobayashi, M. and Koh, T., Earthquake response prediction and rationalization of dynamic design of mid-story isolated buildings. Journal of Structural and Construction Engineering, Transaction of AIJ, (592), pp. 51-57, 2005 (in Japanese).

[7] Yamashita, T., Mukai, Y. and Inoue, Y., Evaluation and synthesis for aseismic responses of structural system with inter-story isolation devices. Journal of Structural and Construction Engineering, Transaction of AIJ, (591), pp. 35-42, 2005 (in Japanese).

[8] Ryan, K.L. and Earl, C.L., Analysis and Design of Inter-Story Isolation Systems with Nonlinear Devices. Journal of Earthquake Engineering, 14(7), pp. 1044-1062, 2010.

[9] Wang, S.J., Chang, K.C., Hwang, J.S. and Lee, B.H. Simplified Analysis of Mid-Story Seismically Isolated Buildings. Earthquake Engineering \& Structural Dynamics, 40(2), pp. 119-133, 2011.

[10] Igusa, T., Der Kiureghian, A. and Sackman, J.L., Modal decomposition method for stationary response of non-classically damped systems. Earthquake Engineering \& Structural Dynamics, 12(1), pp. 121-136, 1984.1

[11] Zhou, X.-Y., Yu, R.-F. and Dong, D., Complex mode superposition algorithm for seismic responses of non-classically damped linear MDOF system, Journal of Earthquake Engineering, 8(4), pp. 597-641, 2004.

[12] Song, J., Chu, Y.-L., Liang, Z. and Lee, G.C., Modal Analysis of Generally Damped Linear Structures Subjected to Seismic Excitations. Report No. MCEER-08-0005, MCEER, Buffalo, NY, 2008. 